\title{
Skin cancer
}

\section{An ideal candidate for preventive medicine}

\author{
Spencer Chambers \\ Faculty Reviewer: Aaron Grant, MD, FRCSC (Department of Plastic and Reconstructive Surgery)
}

\section{ABSTRACT}

Skin cancer is the most common type of neoplasm worldwide. Skin cancer can be classified as non-melanoma skin cancer (NMSC) or melanoma skin cancer (MSC). NMSCs are more common lesions, and typically carry a good prognosis. MSCs are rarer, but cause the majority of skin cancer-related death and morbidity. The biggest causative factor for any skin cancer is ultraviolet radiation exposure (UVR). UVR comes from the sun or synthetic sources such as tanning beds, making it highly avoidable through behavioral change. Despite this, the incidence of skin cancer in Canada has risen over the past decades at an alarming rate. There are many levels of preventive medicine currently in place attempting to change this trend. Primary strategies include wearing hats and protective clothing, as well as avoiding peak daytime hours and tanning beds. At a secondary level, clinical skin exams and public education work to identify disease at earlier stages to make treatments more effective. Both surgical and destructive management can be effective, but success is highly dependent on the stage of disease. Even with appropriate treatment there is a risk of complications including deformity, recurrence and even death. This emphasizes the best treatment for skin cancer is prevention and further underscores the need for behavioural changes at the population level.

\section{INTRODUCTION}

Skin cancers are the most common cancer diagnosed worldwide and affect 80000 Canadians annually. ${ }^{1}$ This is more than breast, colorectal, lung and prostate cancer combined. ${ }^{1}$ While there are different etiologies, the most common cause of cutaneous malignancy is cumulative ultraviolet radiation (UVR) exposure. UVR damage is reported to be responsible for $90 \%$ of all skin cancer diagnoses. ${ }^{2}$ Decreasing exposure to UVR is controllable through simple personal interventions such as sun protection and sun avoidance. ${ }^{2}$ Despite this, the incidence of skin cancer in Canada has increased over the past several decades. ${ }^{1}$ While the majority of these malignancies are low grade lesions that respond well to early treatment, the incidence of more concerning malignancies such as melanomas has also increased..$^{2-4}$ As skin cancer is a prevalent disease with modifiable risk factors, it serves as an ideal candidate for the focus of preventive strategies.

\section{EPIDEMIOLOGY}

Skin cancer is classified as a non-melanoma skin cancer (NMSC) or melanoma skin cancer (MSC). ${ }^{2}$ The majority are
NMSCs, which are comprised of basal cell carcinoma (BCC) and squamous cell carcinoma (SCC). BCCs are the most common, accounting for $80 \%$ of all skin cancer cases. ${ }^{1}$ These lesions usually have a very indolent course, with a less than $0.6 \%$ of cases metastasizing. ${ }^{3}$ Of the numerous subtypes, nodular BCC is the most common and typically arises on the face, neck or ears. Clinical they appearance as a nodular lesion with pearly borders and scattered telangiectasias (Figure 1). ${ }^{2}$ SCCs are less common and account for up to $15 \%$ of skin cancer diagnoses. ${ }^{1}$ Their malignant potential is larger with a $5 \%$ rate of metastasis. ${ }^{5}$ SCCs arise in the same regions as BCCs, and appear as a non-healing ulcer with surrounding erythematous skin (Figure 2). Both types of NMSCs carry a good prognosis, and despite being very common, carry a very low risk of mortality.

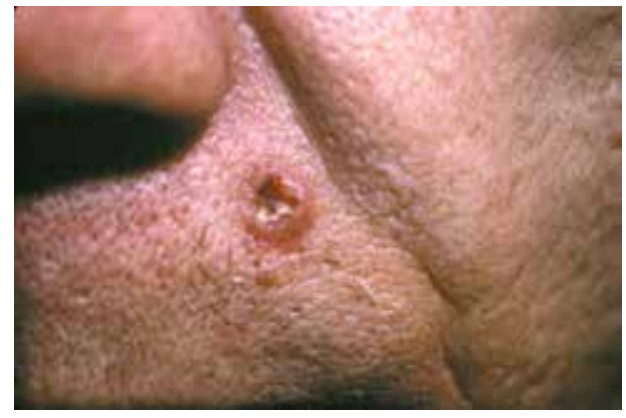

Figure 1. A nodular BCC located in the midface with a characteristic pearly surface and scattered central telangiectasias.

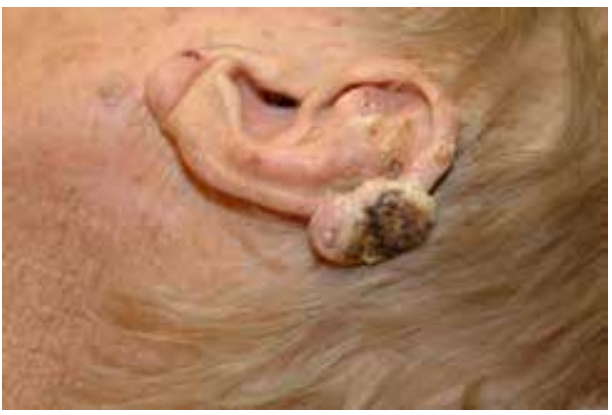

Figure 2. A complicated SCC involving the pinna of the ear. Anteriorly, areas of erythematous scaly skin demonstrating premalignant changes that are characteristic of an actinic keratosis are present.

MSC is a less common but more dangerous form of skin cancer, accounting for less than $5 \%$ of all cases. ${ }^{1}$ The most common subtype of melanoma in Canada is superficial spreading., ${ }^{1,2}$ These lesions present as an asymmetrical flat patch of pigmented skin (Figure 3). Despite their relative rarity, MSCs are responsible for $80 \%$ of all skin cancer-related deaths. ${ }^{5}$ Approximately half of all diagnoses 


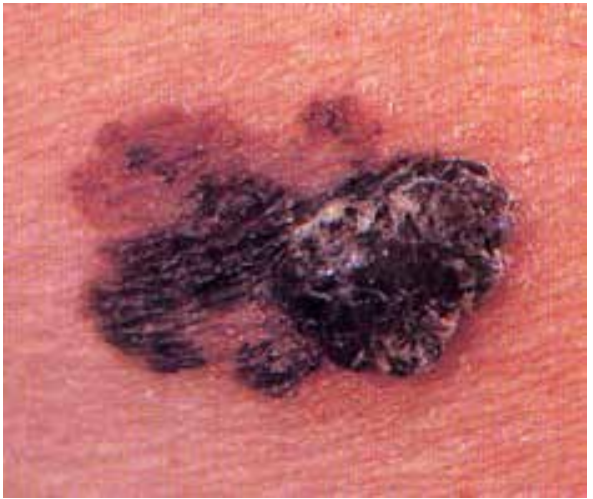

Figure 3. A malignant melanoma exemplifying many screening criteria including asymmetry, irregular boarders, color variegation, diameter $>6 \mathrm{~mm}$, and elevation.

are made in individuals under 60 years old, leading to mortality in young otherwise healthy patients. ${ }^{1}$ Prognosis is related to stage with locally contained melanoma having a 5 -year mortality rate of $3 \%$ or less. ${ }^{7}$ Once MSC disseminates, this prognosis rapidly deteriorates with stage IV MSC having a 5-year mortality in excess of $80 \%$. Even with appropriate treatment, there are high rates of recurrence in patients with late stage disease. ${ }^{8}$ MSC is very aggressive and can metastasize rapidly, making early detection paramount for survival. ${ }^{9}$ Despite the fact that the majority of skin cancers are preventable, the incidence in Canada has increased rapidly over the last 30 years. $^{7}$ Melanoma alone is 20 times more common than it was 60 years ago. ${ }^{10}$ The reasons for this remain unclear and are likely multifactorial, but some proven associations include increased sun exposure and the use of tanning beds. ${ }^{4}$

\section{RISK FACTORS}

Development of skin cancer is based on innate and behavioural risk factors. Innate risk factors are those related to genetics and therefore not easily modifiable. Examples include hereditary skin cancers and fairer skin types. ${ }^{2}$ Although gene modification may be possible in the future, behavioural risks related to environmental exposures should be the current focus of prevention. Modifiable risk factors that play a role in the development of skin cancers include smoking, immunodeficiency, radiation, arsenic exposure, and human papillomavirus (HPV) infection. ${ }^{1,2}$ However, cumulative exposure to UVR is the major contributor. ${ }^{1,2}$ NMSCs are strongly associated with low grade sun exposure that occurs over a prolonged timeline. ${ }^{1,11}$ As skin cells are repeatedly damaged by UVR, the probability of a malignant change occurring increases. This is evident in the association of increasing age and development of NMSCs. ${ }^{2,12,13}$ Melanomas are strongly associated with intense, intermittent exposures. ${ }^{2}$ Blistering sunburns at any age can significantly increase an individual's risk of developing MSC. ${ }^{14}$ Limiting sun exposure in all forms is a vital aspect of prevention of cutaneous malignancy. This can be achieved through the use of sunscreen, safe sun behaviour and the avoidance of UVR exposure during peak hours. ${ }^{5,6}$

\section{PRIMARY PREVENTION}

The simplest way to limit UVR is sun avoidance, this can be achieved by limiting exposure during peak hours. ${ }^{10}$ When avoidance of UVR isn't possible, protective strategies such as appropriate clothing are beneficial. Hats are of particular importance because they protect surfaces that often receive the highest amounts of sun exposure., ${ }^{5,612}$ Protective clothing should also be bolstered with sunscreen. There are many different products, but they all work to prevent harmful UVR from reaching the skin by reflecting or scattering rays. ${ }^{13}$ The ability of these products to stop UVR is measured in terms of a sun protection factor (SPF).$^{13}$ A higher SPF implies that a larger proportion of UVR is being blocked, which extends the amount of time that can be safely spent in the sun. Sunscreen should be worn by anyone over the age of 6 months, and should be applied at least 30 minutes prior to first exposure. ${ }^{13} \mathrm{With}$ prolonged outings, sunscreen should be reapplied at least every 2 hours. ${ }^{13}$ Protective sun behaviours are particularly important in adolescents and teens, who are known to have poor compliance and are more likely to use tanning beds. ${ }^{15}$ Current Canadian initiatives aimed at these generations are focused on the creation and distribution of educational materials to primary and secondary schools. ${ }^{16}$ Other efforts can be seen in the purposed legislature that limit tanning bed use in minors. ${ }^{16}$ Unfortunately, these efforts vary from province to province and there is yet to be a countrywide stand to limit UVR exposure. ${ }^{16}$

In comparison to other developed nations, Canada is lagging behind and would benefit from a unified, national approach against skin cancer.

\section{EARLY DETECTION}

Limiting UVR exposure markedly decreases the chances of developing skin cancer, ${ }^{2,5,13}$ but it does not eliminate all risk. When malignancy does occur, the success of treatment is highly correlated to the time of presentation and stage of disease. ${ }^{2,5}$ Skin exams and monitoring of suspicious lesions allows for the identification of early cancers and premalignant lesions, leading to more effective treatment. Examples of premalignant lesions include actinic keratoses (Figure 3), from which up to $80 \%$ of SCCs arise. ${ }^{2,12}$ Screening is even more important with MSCs because prognosis is directly related to the depth of invasion. ${ }^{2,6}$ Much effort has been invested into the identification of early melanomas, with one of the most practical screening tools being the "ABCDE" mnemonic., ${ }^{2,17}$ Lesions are evaluated for the presence of Asymetry, Border irregularity, Color variegation, Diameter $>6 \mathrm{~mm}$, and Evolution/Elevation/Exposure. These criteria make it possible to identify up to $95 \%$ of MSCs, with the number of features present being correlated to the likelihood of a positive diagnosis. ${ }^{2,17}$ In current time, the ABCDE tool has been employed in numerous apps to be used by patients and primary care providers to assist in MSC diagnosis. ${ }^{18}$ Although these apps show potential, their performance currently is too variable to be used in a clinical setting. ${ }^{18}$ 


\section{EARLY TREATMENT}

If preventive measures have failed and a skin cancer develops, early treatment is recommended. Low grade NMSCs can be treated using a variety of therapies including cryotherapy, curettage and electrodessication, and topical 5-fluorouracil (5-FU), among others. Cryotherapy uses liquid nitrogen to destroy malignant cells through inducing venous stasis and cellular ice crystal formation. ${ }^{18}$ Curettage and electrodessication is a process where tumors are damaged with electricity, then scraped away using a curette. The process is repeated until normal dermis is uncovered. ${ }^{18} 5$-FU is a topical chemotherapeutic agent which interferes with cellular replication leading to neoplastic death. ${ }^{18}$ Each of these treatments can be very effective, with 5 -year cure rates over $92 \%$ in appropriately selected lesions. ${ }^{2,6,18}$

NMSCs that are complex based on location, histology, size or tumor type, as well as all MSCs should be treated with surgical removal. ${ }^{10}$ Approaches include excision with defined margins, frozen section or Mohs microsurgery (MMS). MMS is the gold standard, as it preserves maximal tissue while boasting a 5-year cure rate of $97 \%$. However, MMS is also the most expensive and time-consuming. For these reasons, it is typically reserved for aggressive tumors or recurrent neoplasms in sensitive locations. ${ }^{18}$ Surgical excision with defined margins is more commonly used, as it balances resources and outcomes. This procedure has 5-year cure rates ranging from $92 \%$ to $95 \%$ and requires much less time and fewer resources. ${ }^{17}$ Regardless of modality, there is no treatment method that is perfect. Although morbidity and mortality can be improved, ${ }^{10}$ there is always a risk of recurrence and complications.

\section{CONCLUSION}

Although BCCs, SCCs and MSCs are unique diseases with different epidemiology, pathophysiology, presentation, and natural history, they share a common modifiable risk factor: UVR. ${ }^{1,2,11,12}$ Decreasing UVR exposure is an achievable and effective intervention that should be practiced by patients of all ages. Patient education and awareness about skin cancer and the associated risks can lead to simple behavioural changes that may reduce the risk of skin cancer. $^{2,5}$

\section{REFERENCES}

1. Canadian Cancer Society's Advisory Committee on Cancer Statistics. Canadian Cancer Statistics 2015. Toronto, ON: Canadian Cancer Society; 2015.

2. Throne HC, Chung KC, Gosain AK, et al. Grabb and Smiths Plastic Surgery. 7th rev., Philadelphia: Lipinkott Williams and Wilkins; 2014. $1050 \mathrm{p}$.

3. Ganti AK, Kessinger A. Systemic therapy for disseminated basal cell carcinoma: An uncommon manifestation of a common cancer. Cancer Treat Rev. 2011 Oct;37(6):440-3.

4. Canadian Cancer Society. Melanoma: deadliest type of skin cancer is on the rise. [Internet] Canadian Cancer Society; 2014 [cited 2016 Apr] Available from: https://www.cancer.ca/en/about-us/for-media/ media-releases/national/2014/2014-canadian-cancer-statistics/?region $=$ on
5. Scottish Intercollegiate Guidelines Network (SIGN). Management of primary cutaneous squamous cell carcinoma. Edinburgh: SIGN; 2014. [cited 2016 Apr] Available from: http://www.sign.ac.uk

6. Holterhues AM, Hollestein C, Coebergh JW, et al. Trends in incidence and predictions of cutaneous melanoma across Europe up to 2015. J Eur Acad Dermatol Venereol. 2014 Sep;28(9):1170-8.

7. American Cancer Society. Melanoma Skin Cancer.[Internet] American Cancer Society; 2016. [cited 2016 Apr] Available from: http://www. cancer.org/cancer/skincancer-melanoma/detailedguide/melanoma-skin-cancer-survival-rates

8. Tas F. Metastatic Behaviour in Melanoma: Timing, Pattern, Survival, and Influencing Factors. J Oncol. 2012; Volume 2012.

9. Rigel DS, Robinson JK, Ross MI, et al. Cancer of the Skin: Expert Consult. Philadelphia, Pa: Elsevier Saunders; 2005.

10. Gies P. Photoprotection by clothing. Photodermatol Photoimmunol Photomed 2007;23:264-74.

11. Alam M, Ratner D. Cutaneous squamous cell carcinoma. N Engl J Med. 344:2001;975-83.

12. Howard WR, Weinstock MA, Harris AR, et al. Non-melanoma skin cancer in the United States: Incidence. J Am Acad Dermatol. 30:1994;774-8.

13. Dennis LK, Vanbeek M J, Freeman LE, et al. Sunburns and risk of cutaneous melanoma: does age matter? A comprehensive meta-analysis. Ann Epidemiol. 2008;18(8):614-27.

14. Skin Cancer Foundation "Sunscreens Explained" Skin Cancer Foundation; 2012. [cited $2016 \mathrm{Apr}$ ] Available from: http://www.skincancer org/prevention/sun-protection/sunscreen/sunscreens-explained

15. Joshua AM. Melanoma prevention: are we doing enough? A Canadian perspective. Curr Oncol. 2012 Dec; 19(6): e462-7.

16. American Academy of Dermatology Ad Hoc Task Force for the ABCDEs of Melanoma. Early detection of melanoma: reviewing the ABCDEs. J Am Acad Dermatol. 2015 Apr;72(4):717-23.

17. Wolf JA, Moreau JF, Akilov O. Diagnostic Inaccuracy of Smartphone Applications for Melanoma Detection. JAMA Dermatol. 2013;149(4):422-6. 